\title{
Modification of the electronic transport in Au by prototypical impurities and interlayers
}

\author{
M. M. FAdlallah ${ }^{1,2}$, C. Schuster ${ }^{1}$, U. ECKERN ${ }^{1}$ and U. Schwingenschlögl ${ }^{3(a)}$ \\ ${ }^{1}$ Universität Augsburg, Institut für Physik - 86135 Augsburg, Germany, EU \\ ${ }^{2}$ Benha University, Physics Department - Benha, Egypt \\ ${ }^{3}$ KAUST, PSE Division - Thuwal 23955-6900, Kingdom of Saudi Arabia
}

PACS 73.20. $-r$ - Electron states at surfaces and interfaces

PACS 73.40.-c - Electronic transport in interface structures

\begin{abstract}
Electronic transport calculations for metallic interfaces based on density functional theory and a scattering theory on the Landauer-Büttiker level are presented. We study the modifications of the transport through Au due to prototypical impurities and interlayers. Our results show that the influence of $\mathrm{S}$ and $\mathrm{Si}$ impurities is well described in terms of simple vacancies. Metallic impurities and interlayers, on the other hand, have even more drastic effects, in particular when the Au $s$ - $d$ hybrid states at the Fermi energy are perturbed. The effects of a possible interface alloy formation are discussed in detail.
\end{abstract}

Introduction. - In recent years, interfaces have been investigated intensively because they can modify the transport properties drastically compared to the corresponding bulk compound. For example, the charge redistribution at metal-superconductor interfaces imposes severe restrictions on the critical current in wire and tape applications of high-temperature superconductors $[1,2]$. Interfaces are likewise highly important in semiconductor physics. A review on recent developments has been given by Ahn et al. [3]; these include the creation of a highly conductive two-dimensional electron gas at the interfaces between insulators [4-6] and the induced charge density in organic-inorganic devices [7-9].

The electron transport across metallic multilayers is of particular importance for understanding the giant magnetoresistance in ferromagnetic heterostructures, like $\mathrm{Fe} / \mathrm{Cr}$ multilayers $[10,11]$. Also, hot-electron scattering in $\mathrm{Au} / \mathrm{Fe} / \mathrm{Au}$ trilayers is affected by the metal-metal interfaces [12]. In particular, the attenuation of the electrons strongly depends on the thickness of the Fe interlayer. Regarding non-magnetic systems, the properties of metalmetal interfaces, for instance, are of interest in many metallurgical applications [13]. For $\mathrm{Co} / \mathrm{Cu}, \mathrm{Fe} / \mathrm{Cr}$, and $\mathrm{Au} / \mathrm{Ag}$ multilayers the resistance can be increased or decreased by interface disorder, depending on the specific system [14]. The orientation-dependent transparency of

\footnotetext{
(a) E-mail: udo.schwingenschlogl@kaust .edu.sa
}

simple metals, like $\mathrm{Al}$ and $\mathrm{Ag}$, has been investigated in ref. [15]. In addition, simple metal interfaces have recently been studied within transport calculations [16].

In this context, the present study develops a comprehensive picture of the modifications of the charge transport in Au due to the incorporation of prototypical impurities and interlayers. We start our considerations by giving details on the applied computational method and the underlying structural setup in the next section. The results of transport calculations for metallic and non-metallic impurities as well as metallic interlayers are presented in the third section. The thickness dependence of the conductance in multilayer systems as well as the effects of alloy-like interfaces and two-component interlayers on the transmission coefficient are discussed in the fourth section. The conclusions are summarized in the last section.

Computational method and structural setup. In our calculations we apply the SMEAGOL transport code [17], which makes use of the SIESTA program package [18]. SIESTA is an efficient implementation of density functional theory and provides the SMEAGOL code with a self-consistent solution of the equilibrium electronic structure (using periodic boundary conditions). SMEAGOL then calculates the transport properties using a scattering theory on the Landauer-Büttiker level. The valence electrons are described by a single-zeta basis set of linear combinations of numerically adjusted 


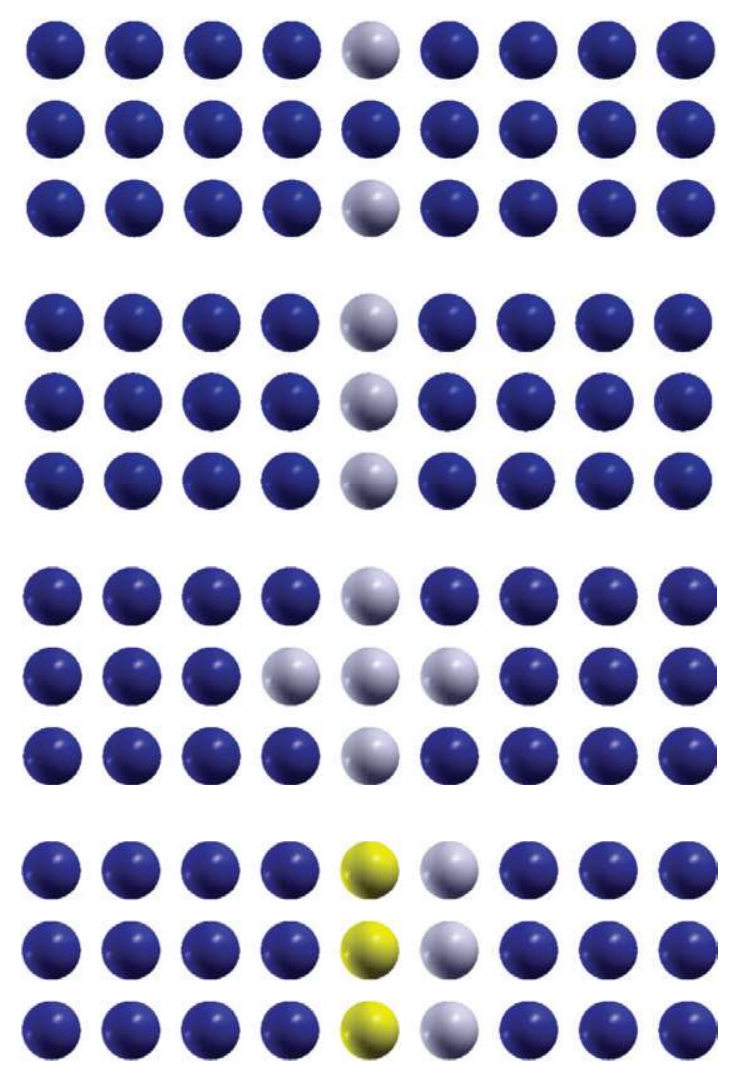

Fig. 1: (Color online) Structures under investigation (from top to bottom): Interface with impurity, metallic interlayer, interface alloy, and two-component interlayer. Blue, gray, and yellow spheres indicate $\mathrm{Au}, \mathrm{Ag}$, and $\mathrm{Cu}$ atoms, respectively.

atom-centered orbitals. We have checked for bulk Au and for the $\mathrm{Cu}$ impurities and interlayers in $\mathrm{Au}$ that deviations between a single- and a double-zeta basis are subordinate. Furthermore, the core electrons are represented by norm-conserving pseudopotentials in the fully non-local form [19] and the exchange-correlation functional is treated in the local density approximation (LDA). The principal layer of the leads comprises two $\mathrm{Au}$ unit cells and the scattering region consists of six fcc unit cells in each case. We apply periodic boundary conditions perpendicular to the [100] transport direction, where the periodic unit is one fcc unit cell. For the leads, we employ a $15 \times 15 \times 100$ Monkhorst-Pack $k$-grid for sampling the Brillouin zone, while the $k$-grid is $10 \times 10 \times 1$ for the device region. We study the electronic transport through interfaces oriented perpendicular to the transport direction.

Impurity systems are generated by replacing each second interface atom by an impurity atom $(\mathrm{Mg}, \mathrm{Si}, \mathrm{S}$, $\mathrm{Ni}$, and $\mathrm{Ag}$ ), see the top structure in fig. 1. The interlayer systems, on the contrary, consist of impurity monolayers $(\mathrm{Cu}$ and $\mathrm{Ag})$ in the center of the $\mathrm{Au}$ host. In addition, we consider the formation of an interface alloy [20] and heterostructures with two-component interlayers. The interface alloy is modeled as an impurity monolayer with additional impurities on both sides. The different setups

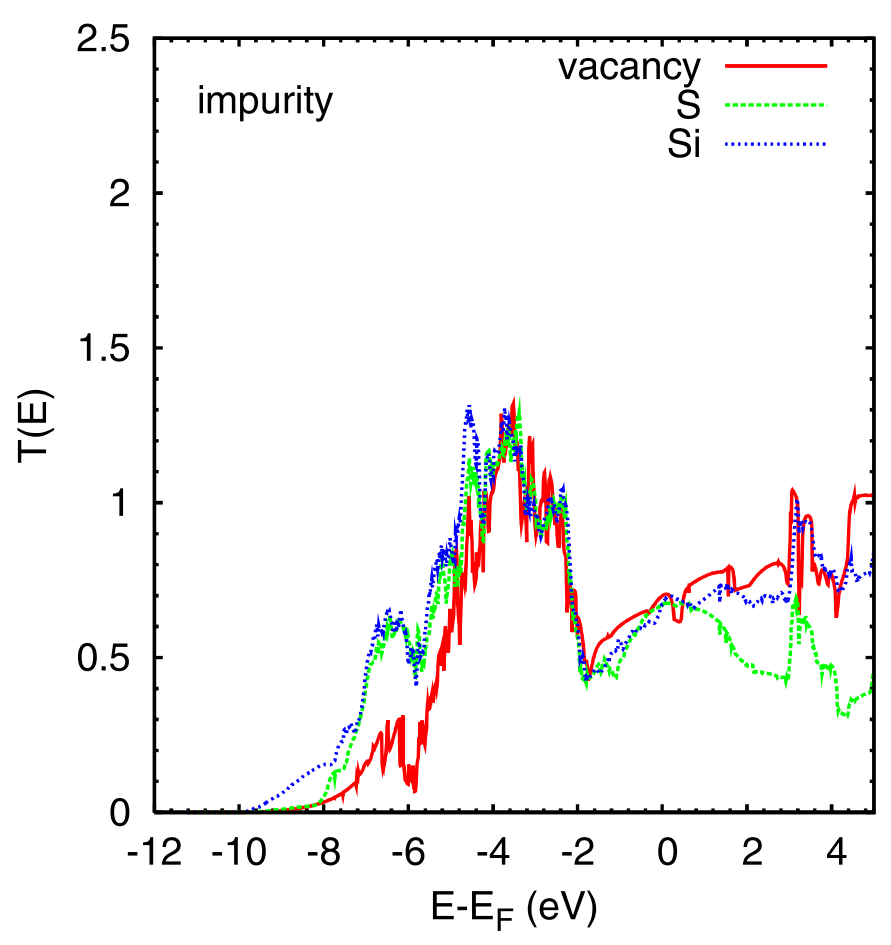

Fig. 2: (Color online) Transmission coefficient of the interfaces with $\mathrm{S}$ and Si impurities, as compared to the interface with a vacancy.

are illustrated in fig. 1. We also address the dependence of the conductance on the interlayer thickness. In order to highlight the principal effects induced by our prototypical interface modifications, we do not take into account any additional structure relaxation.

Impurities and interlayers. - In the first step we compare the effects of non-metallic and metallic impurities. As non-metallic impurities we consider Si (electronic configuration $3 s^{2} 3 p^{2}$, chemically inert) and the chalcogen $\mathrm{S}$ (electronic configuration $3 s^{2} 3 p^{4}$ ) which are typical impurites found in $\mathrm{Au}$. Crystalline $\mathrm{Si}$ and $\mathrm{S}$ are insulators. In fig. 2 we compare the effects of $\mathrm{Si}$ and $\mathrm{S}$ impurities on the transmission coefficient $T(E)$ and also show the corresponding data for a vacancy system taken from ref. [16]. Here and in all following figures, $T(E)$ is normalized with respect to the number of points in the $k$-mesh. The (zerobias) conductance $G=G_{0} \cdot T\left(E_{F}\right)$ obtained for the three systems shows rather similar values close to $G=0.6 \cdot G_{0}$, where $G_{0}=2 e^{2} / h$. Just below the Fermi energy $E_{F}$ the transmission coefficient grows almost linearly with the energy, reflecting a delocalized nature of the related states. In addition, the reduction of the transmission due to the impurities in the energy range from $-6 \mathrm{eV}$ to $-2 \mathrm{eV}$, where the $\mathrm{Au} 5 d$ states dominate, is similar in all three cases. Since the $d$ bands are more sensitive to local disorder [21], the reduction here is much stronger than near $E_{F}$. Minor differences between the three systems in fig. 2 concern the lower band edge. Below $-8 \mathrm{eV}(s$-like $\mathrm{Au}) T(E)$ is suppressed for both the S impurity and the vacancy but not for the Si impurity, because Si states are present in this 


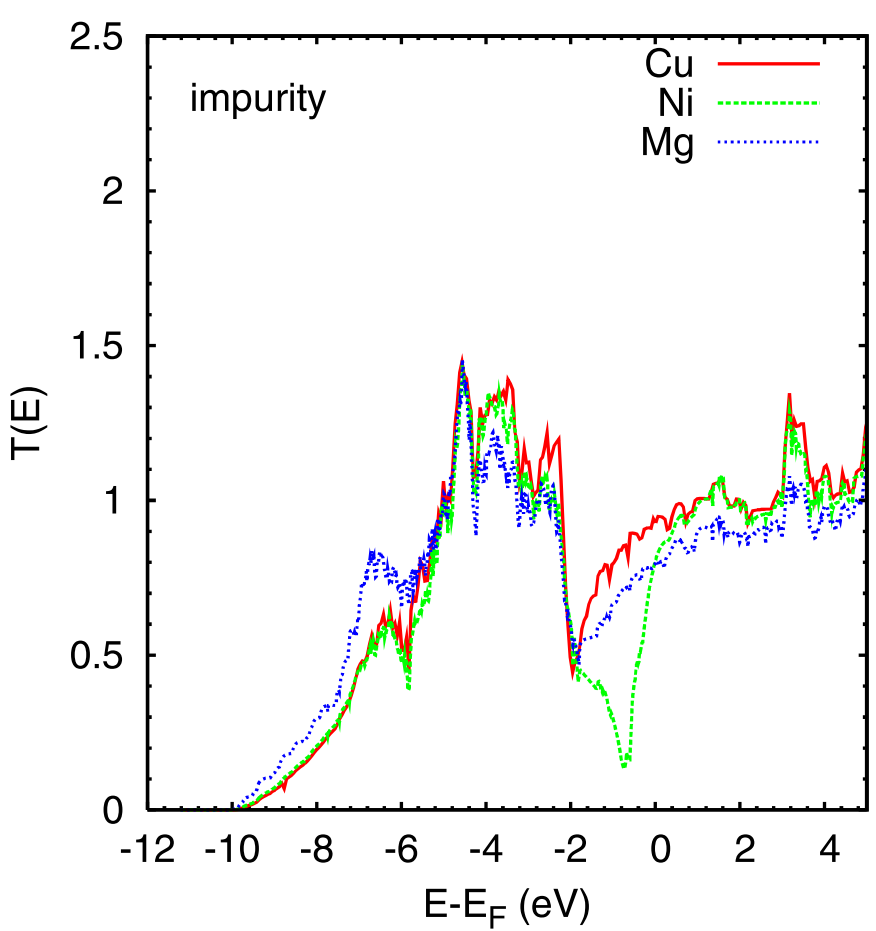

Fig. 3: (Color online) Transmission coefficient of the interfaces with $\mathrm{Cu}, \mathrm{Ni}$, and $\mathrm{Mg}$ impurities.

energy range and hybridize with the $\mathrm{Au}$. Between $-8 \mathrm{eV}$ and $-6 \mathrm{eV}$ ( $s$ - and $d$-like $\mathrm{Au}$ ) the impurities do not reproduce the reduction of $T(E)$ as found for vacancies. Apart from that, non-metallic impurities resemble the effects of vacancies even quantitatively.

Turning to the metallic impurities $\mathrm{Mg}$ and Ni, a comparison to previous findings for a $\mathrm{Cu}$ impurity [16] is helpful. $\mathrm{Mg}$ is a metal which can be described by a nearly free electron model. $\mathrm{Ni}$ and $\mathrm{Cu}$, on the other hand, are transition metals with narrow $d$-bands. Despite this different physical nature of the three metals, they behave rather similar when acting as impurities in $\mathrm{Au}$, see fig. 3. The onset of $T(E)$ is found at $-9.5 \mathrm{eV}$ and the conductance is $\sim 0.8 \cdot G_{0}$. Also, the reduction of $T(E)$ in the $\mathrm{Au} 5 d$ dominated energy range is comparable. However, a pronounced reduction of $T(E)$ near $-0.5 \mathrm{eV}$ is obtained for the $\mathrm{Ni}$ impurity. We may conjecture that $\mathrm{Au}-\mathrm{Ni}$ bonding, in contrast to $\mathrm{Au}-\mathrm{Cu}$ bonding, affects the $\mathrm{Au} s$ - $d$ hybrid states. In fact, the $\mathrm{Ni}$ $3 d$ states are located around $-1 \mathrm{eV}[22]$, i.e. in the region of the $\mathrm{Au} s$ - $d$ hybrid states, whereas the $\mathrm{Cu} 3 d$ states are located around $-2 \mathrm{eV}$, i.e. in the $\mathrm{Au} 5 d$ dominated energy range. Hence, only the Ni $3 d$ states can interact with the Au $s$ - $d$ hybrid states and form a $d$ - $d$ hybrid. The $\mathrm{Au} 6 s$ states, in turn, hybridize with the $\mathrm{Ni} 4 s$ states and both shift to much lower energy. Since the Ni $4 s$ states therefore are missing in the vicinity of the Fermi energy, the transmission is strongly reduced. We find the highest conductivity for the $\mathrm{Cu}$ impurity system, $T\left(E_{F}\right)=0.93$, since in this case the electronic structure of the impurity is closely related to that of the $\mathrm{Au}$ host. For the $\mathrm{Mg}$ system the states deviate more while the impurity is still

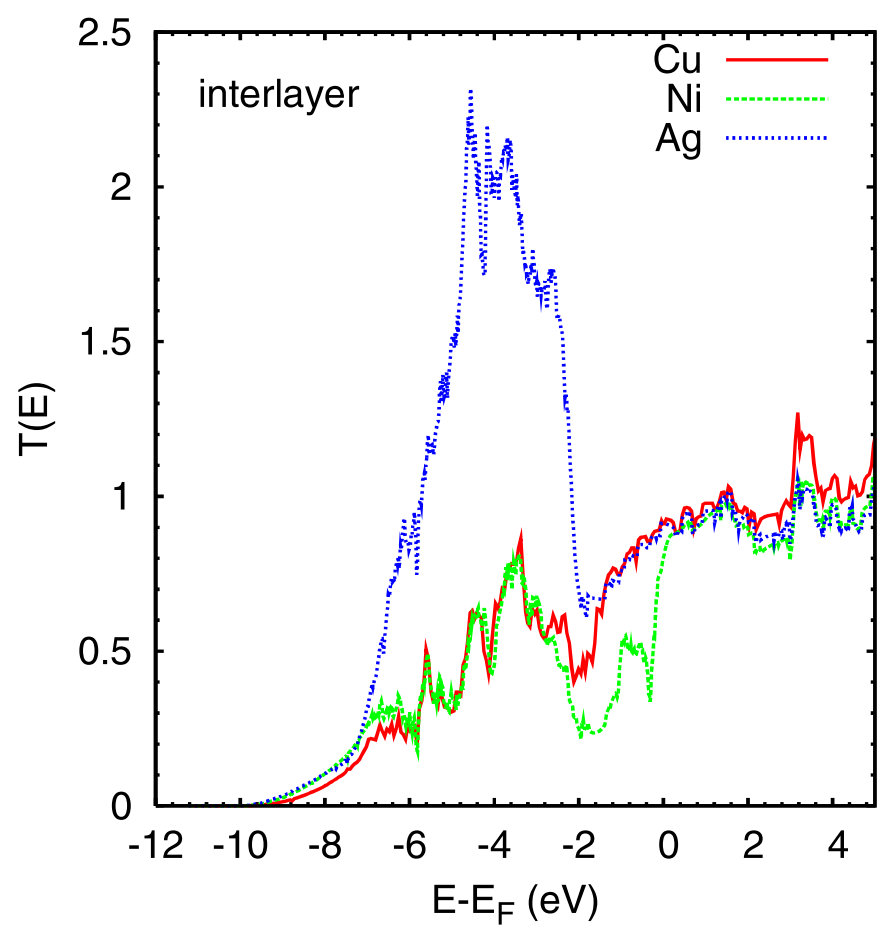

Fig. 4: (Color online) Transmission coefficient of the $\mathrm{Cu}, \mathrm{Ni}$, and $\mathrm{Ag}$ interlayer systems.

metallic, which results in a reduction of the conductivity, $T\left(E_{F}\right)=0.78$. A further reduction is expected in the case of a non-metallic impurity. An example is the $\mathrm{S}$ system for which we obtain $T\left(E_{F}\right)=0.63$.

Going beyond the impurity systems, we next study full impurity interlayers of $\mathrm{Cu}, \mathrm{Ni}$, and $\mathrm{Ag}$, which all crystallize in a fcc structure. In addition, the lattice constant and valence electronic structure of $\mathrm{Ag}$ are rather similar to Au. As discussed in detail in ref. [16], the transmission mediated by the $\mathrm{Au} 5 d$ states usually is smaller in interlayer than in impurity systems, while both the onset of $T(E)$ and the conductance agree very well. The transmission coefficients of $\mathrm{Cu}, \mathrm{Ni}$, and $\mathrm{Ag}$ interlayer systems are depicted in fig. 4. In order to understand the high values of $T(E)$ in the $\mathrm{Ag}$ case, as compared to $\mathrm{Cu}$ and $\mathrm{Ni}$, we address the related densities of states (DOS): Since the $\mathrm{Cu}$ and $\mathrm{Ni} 3 d$ bands are very narrow, they do not hybridize with the Au states and the Au $5 d$ transmission consequently is significantly reduced. In contrast, Ag has $4 d$ states in the whole energy range between $-8 \mathrm{eV}$ and $-2 \mathrm{eV}$ and therefore strongly hybridizes with the Au host. Furthermore, the reduced transmission between $-2 \mathrm{eV}$ and the Fermi level in the case of the $\mathrm{Ni}$ interlayer system has the same origin as discussed before for the $\mathrm{Ni}$ impurity.

Multilayers and interface alloys. - In this section we deal with modifications of the interlayer system. First, we discuss the influence of an increasing interlayer thickness. Second, we consider interlayers which are not 


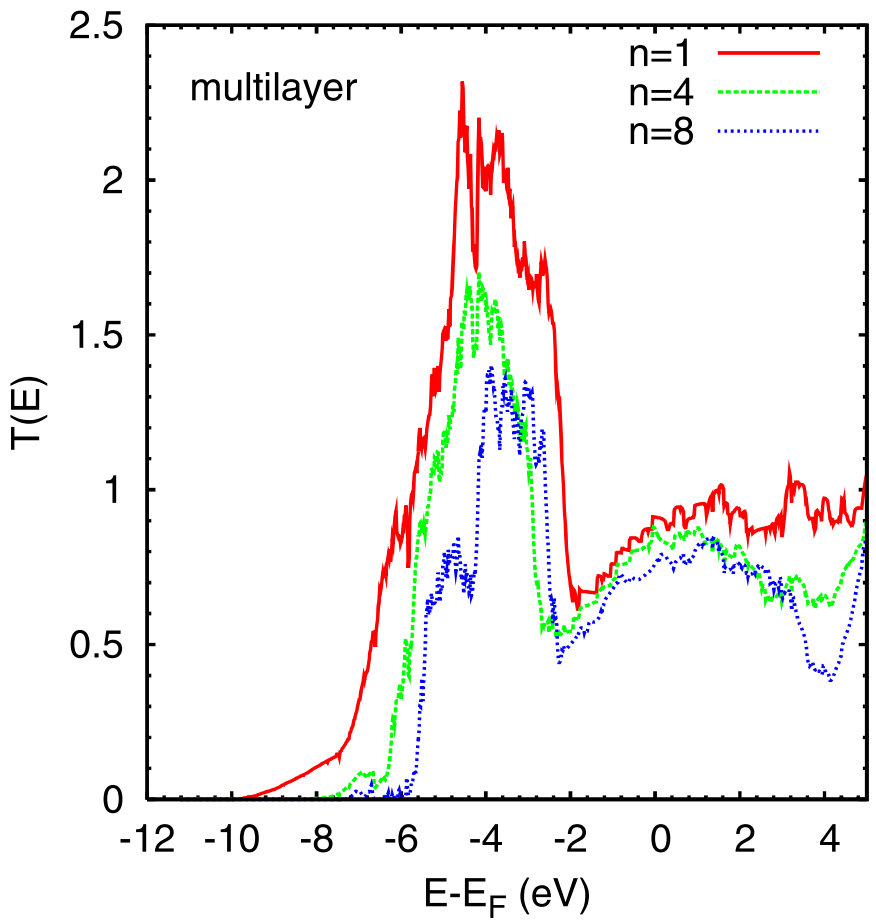

Fig. 5: (Color online) Transmission coefficient of the Ag interlayer system for interlayer thicknesses of $n=1,4$, and 8 .

atomically sharp but show interface alloy formation. Third, we investigate two-component interlayers.

The influence of the interlayer thickness is studied for $\mathrm{Au} / n \mathrm{Ag} / \mathrm{Au}$ heterostructures with $n \mathrm{Ag}$ monolayers. According to fig. 5, the conductance of the system decreases with growing $n$, where no saturation is found up to $n=8$. In addition, the shape of $T(E)$ is closely related to the shape of the bulk DOS of the two components. In the case of a single interlayer, $T(E)$ resembles the DOS of the $\mathrm{Au}$ host. Therefore, a linear increase of $T(E)$ between $-10 \mathrm{eV}$ and $-8 \mathrm{eV}$ ( $\mathrm{Au} s$ states) and a high transmission in the energy range of the $\mathrm{Au} 5 d$ states are observed. With increasing $n$, however, the shape of $T(E)$ is given by the bulk Ag DOS. Since the width of the bulk Ag $4 d$ bands $(\sim 3.5 \mathrm{eV})$ is smaller than the width of the $\mathrm{Au} 5 d$ bands $(\sim 6 \mathrm{eV})$, the broad peak in $T(E)$ narrows with growing $n$. In fact, for $n=8$ the bulk $\mathrm{Ag} 4 d$ band width is reached. At the transmission maximum around $-4 \mathrm{eV}$ the amplitude is suppressed strongly for growing $n$ because of the localized nature of the $d$ states. On the contrary, the reduction of the conductance is much less pronounced, amounting to $4 \%$ at $n=4$ and $16 \%$ at $n=8$ with respect to the $n=1$ value. Finally, we note that these results are specific for the $\mathrm{Au} / n \mathrm{Ag} / \mathrm{Au}$ system and may change with the electronic structures of the component materials.

It is known that alloys can be formed at metallic interfaces [20]. We address this issue by comparing the $n=2$ interlayer system to a configuration with alloy layers next to a single Ag interlayer, see the third structure of fig. 1. Both systems comprise the same number of $\mathrm{Ag}$ atoms. According to fig. 6, the transmission is slightly reduced as compared to the $n=1$ curve, which traces

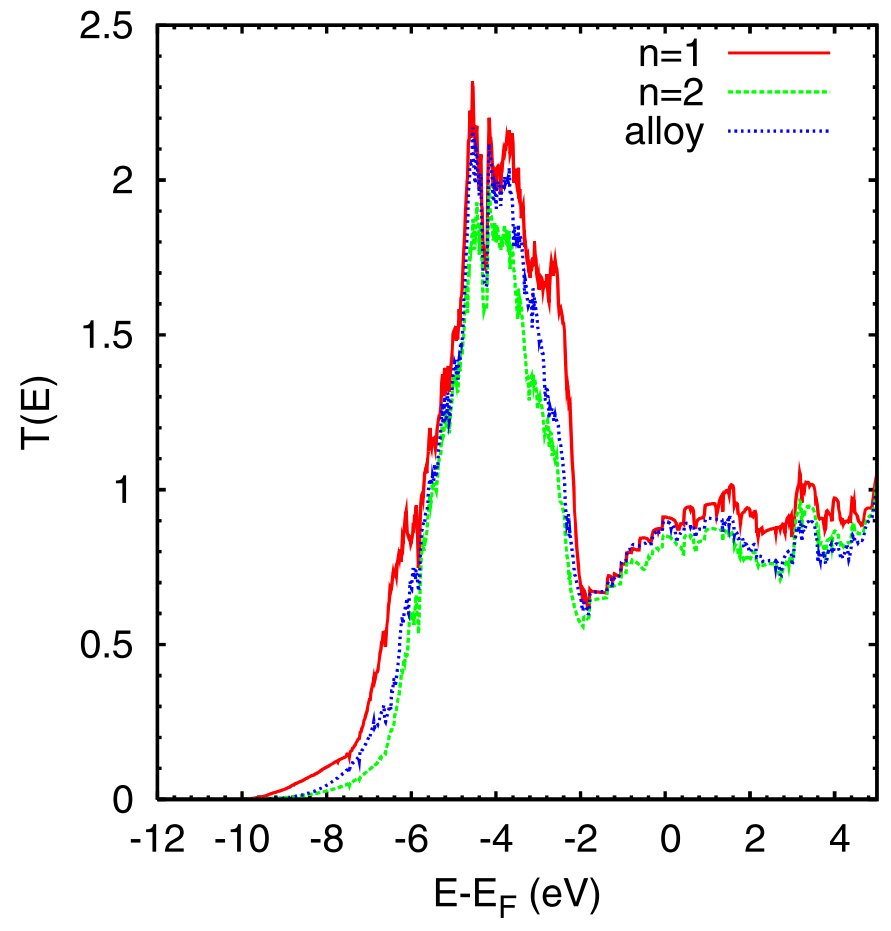

Fig. 6: (Color online) Transmission coefficient of the interface alloy, as compared to the $n=1$ and $n=2$ interlayer systems.

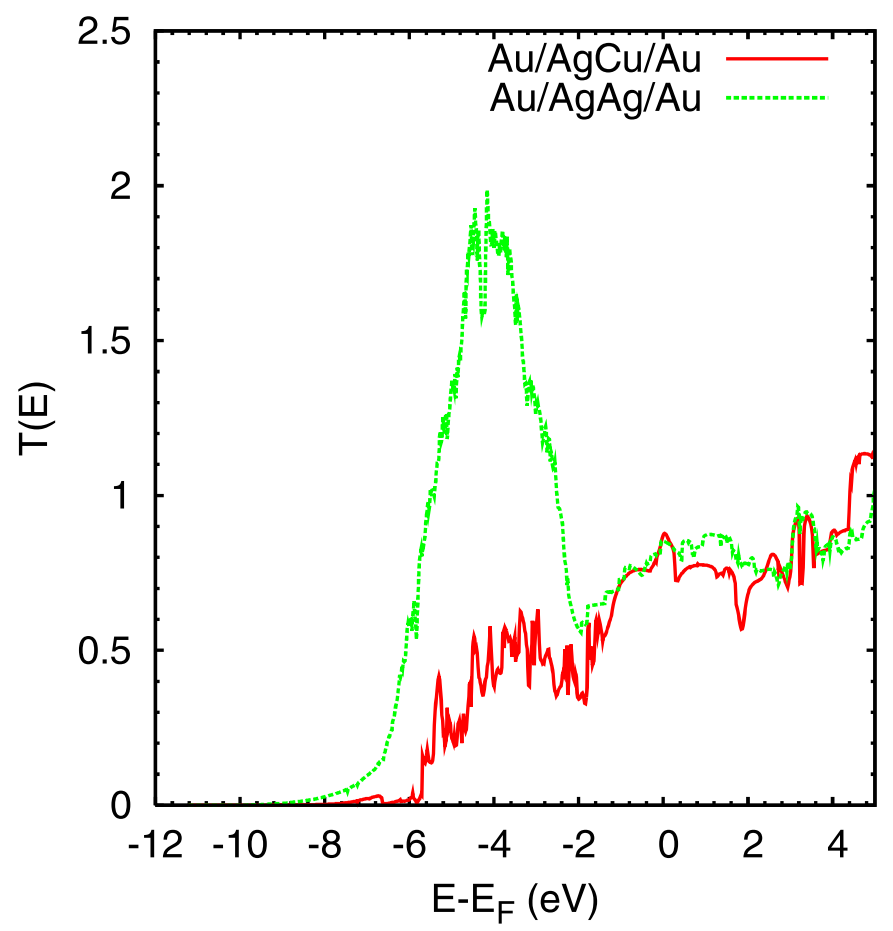

Fig. 7: (Color online) Transmission coefficient of the heterostructure, as compared to the $n=2$ interlayer system.

back to the additional impurities, and, analogously, is higher than for a double Ag layer. Moreover, it is worth mentioning that the alloy formation affects $T(E)$ almost uniformly in the studied energy range.

As seen before, the electronic structure of the impurity/interlayer material strongly influences $T(E)$. This 
becomes even more critical when multiple interlayers of different materials are considered, as we shall demonstrate for the $\mathrm{Au} / \mathrm{AgCu} / \mathrm{Au}$ heterostructure, which we compare to the $\mathrm{Au} / \mathrm{AgAg} / \mathrm{Au}$ heterostructure in fig. 7. Since the conductance is reduced neither for the $\mathrm{Au} / \mathrm{AgAg} / \mathrm{Au}$ heterostructure nor for a single $\mathrm{Cu}$ interlayer, see fig. 4 , it is not surprising that the conductance of the twocomponent interlayer system also resembles the $\mathrm{Au}$ host. The suppression of the transmission below $-2 \mathrm{eV}$ is due to the narrow $\mathrm{Cu} 3 d$ bands, see our earlier discussion. It is even larger than for a single $\mathrm{Cu}$ interlayer, since the $\mathrm{Ag}-\mathrm{Cu}$ interface results in a further localization of these states. The shape of $T(E)$ of the two-component interlayer system again can be interpreted as a superposition of the component effects.

Conclusion. - In conclusion, our results demonstrate that non-metallic impurties alter the electronic transport similar to simple vacancies, since they do not contribute to the electronic states at the Fermi energy. In the case that an impurity, interlayer, or interface alloy resembles the $\mathrm{Au}$ electronic structure, its incorporation alters the transmission only marginally. However, when distinct bonds are formed between the contaminant and the $\mathrm{Au}$ host the transmission can be strongly affected. For Ni impurities, the Au $s$ - $d$ hybridization is suppressed in the vicinity of the Fermi energy due to the formation of $d-d$ hybrid states. The transmission coefficient thus decreases by a factor of four. We find only a minor interdependence between multiple modifications, i.e. their cumulative effect is well described in terms of a superposition of the individual effects. This paves the way for tailoring the energy dependence of the transmission coefficient.

We thank I. Rungger, S. SAnvito, and P. SchwaB for fruitful discussions as well as the Deutsche Forschungsgemeinschaft (SFB 484) and the Egyptian Missions System for financial support.

\section{REFERENCES}

[1] Hilgenkamp H. and Mannhart J., Rev. Mod. Phys., 74 (2003) 485.

[2] Schwingenschlögl U. and Schuster C., Appl. Phys. Lett., 90 (2007) 192502; Phys. Rev. B, 79 (2009) 092505.
[3] Ahn C. H., Bhattacharya A., Di Ventra M., Echstein J. N., Frisbie C. D., Gershenson M. E., Goldman A. M., Inoue I. H., Mannhart J., Millis A. J., Morpurgo A. F., Natelson D. and Triscone J.-M., Rev. Mod. Phys., 78 (2006) 1185.

[4] Окамото S. and Millis A. J., Nature (London), 428 (2004) 630.

[5] Thiel S., Hammerl G., Schmehl A., Schneider C. W. and Mannhart J., Science, 313 (2006) 1942.

[6] Schwingenschlögl U. and Schuster C., EPL, 81 (2008) 17007; 86 (2009) 27005.

[7] Scheffler M. and Stampfl C., in Electronic Structure: Theory and Practical Methods (Cambridge University Press, Cambridge) 2004.

[8] Bagus P. S., Hermann K. and Wöll C. J., J. Chem. Phys., 123 (2005) 184109.

[9] Schuster C. and Schwingenschlögl U., Chem. Phys. Lett., 468 (2009) 75.

[10] Baibich M. N., Broto J. M., Fert A., Nguyen Van Dau F., Petroff F., Etienne P., Creuzet G., Friederich A. and Chazelas J., Phys. Rev. Lett., 61 (1988) 2472.

[11] Binasch G., Grünberg P., Saurenbach F. and Zinn W., Phys. Rev. B, 39 (1989) 2428.

[12] Armour P., Shen T.-H., Ke M.-L. and Grey R., Appl. Surf. Sci., 123-124 (1998) 412.

[13] Yaniv A., Phys. Rev. B, 17 (1977) 3904.

[14] Xu P. X., Kelly P. J., Bauer G. E. W., Turek I., Kudrnovský J. and Drchal V., Phys. Rev. B, 63 (2001) 064407 .

[15] Xu P. X., Zwierzycki M., Talanana M. and Kelly P. J., Phys. Rev. Lett., 96 (2006) 176602.

[16] Fadlallah M. M., Schuster C., Schwingenschlögl U., Wunderlich T. and Sanvito S., J. Phys.: Condens. Matter, 21 (2009) 315001.

[17] Rocha A. R., Garcia-Suarez V. M., Bailey S. W., Lambert C. J., Ferrer J. and Sanvito S., Nat. Mater., 4 (2005) 335; Phys. Rev. B, 73 (2006) 085414.

[18] Soler J. M., Artacho E., Gale J. D., García A., Junquera J., Ordejón P. and SÁnchez-Portal D., J. Phys.: Condens. Matter, 14 (2002) 2745.

[19] Kleinman L. and Bylander D. M., Phys. Rev. Lett., 48 (1982) 1425

[20] Santra A. K. and Rao C. N. R., Appl. Surf. Sci., 84 (1995) 347.

[21] Autés G., Barreteau C., Spanjaard D. and Desjonquéres M.-C., Phys. Rev. B, 77 (2008) 155437.

[22] dos Santos V. and Kuhnen C. A., Thin Solid Films, 350 (1999) 258. 